\title{
Workspace and Dexterity Investigation of the Master Robot in Sina flex Robotic Telesurgery System: An in Vivo Validation
}

This paper was downloaded from TechRxiv (https://www.techrxiv.org).

\section{LICENSE}

CC BY 4.0

SUBMISSION DATE / POSTED DATE

26-02-2022 / 03-03-2022

\section{CITATION}

Aghanouri, Mehrnaz; Moradi, Hamid; Alibeik, Hossein Arab; Mirbagheri, Alireza (2022): Workspace and Dexterity Investigation of the Master Robot in Sina flex Robotic Telesurgery System: An in Vivo Validation. TechRxiv. Preprint. https://doi.org/10.36227/techrxiv.19242516.v1

$\mathrm{DOI}$ 


\title{
Workspace and Dexterity Investigation of the Master Robot in Sina flex Robotic Telesurgery System: An in Vivo Validation
}

\author{
Mehrnaz Aghanouri, Student Member, IEEE, Hamid Moradi, Hossein A. Alibeik, and Alireza \\ Mirbagheri
}

\begin{abstract}
Robotic surgery technique has gained admissibility in recent years. To provide ergonomic postures for the surgeon along with dexterous workspace for the Sinaflex robotic telesurgery system, a new haptic interface is introduced at the surgeon side of the robot. To analyze its performance, in this paper, the forward and inverse kinematics of the master manipulator are derived using a new geometrical approach. The workspace of the robot is derived, and its dexterity is investigated. Finally, to show the operational workspace and dexterity, the data collected during a dogs' vasectomy robotic surgery by Sina flex are analyzed. According to the simulation results, the workspace of the end effector is as large as $600 \times 571 \times 560 \mathrm{~mm}^{3}$ which is 40 times greater than the ergonomic human hand workspace. The in-vivo experiment shows that the operational surgery workspace is about $2.6 \%$ and $3.4 \%$ of the whole workspace for the left and right master manipulator, respectively. The dexterity of the robot based on the Global Conditioning Index, during simulation and experiment, for the position is 0.4557 and 0.6565 , respectively. A large workspace along with its high dexterity of the master manipulator of Sinaflex robotic telesurgery system make it a good choice to fulfill robotic surgery technique requirements.
\end{abstract}

\section{INTRODUCTION}

Robotic surgery is one of the most obvious effects of technology in healthcare improvements. This technology was introduced to medical service in the late 1980s for the orthopedic aims [1]. Proving its advantages over the open surgery and laparoscopy technique such as less recovery time, less surgery complications, increasing accuracy, and providing an ergonomic posture for the surgeons, makes it the first choice for gynecology, gastroenterology and urology surgeries [2, 3]. Robotic surgery systems are composed of the surgeon side and patient side subsystems. To fulfill the surgeon desires to access to all points of the surgery sites, a good master robot should provide three important characteristics: enough degree of freedom (DoF), a large enough workspace, and high dexterity $[4,5]$. In this regard, various master robots or haptic interfaces are developed [6]. The mechanisms of these robots can be categorized to serial,

M. Aghanouri, and H. Moradi, are with the Department of Medical Physics and Biomedical Engineering, School of Medicine, Tehran University of Medical Sciences, Tehran, Iran (e-mail: maghanouri@razi.tums.ac.ir).

H. A, Alibeik, and A. Mirbagheri, are with the Department of Medical Physics and Biomedical Engineering, School of Medicine and joint affiliated with Research Center for Biomedical Technologies and Robotics (RCBTR), Advanced Medical Technologies and Equipment Institute (AMTEI), Tehran University of Medical Sciences (TUMS), Tehran, Iran. (e-mail: arabalibeik@tums.ac.ir), (phone: 0098-21-64053245; fax: 0098-2166482654; e-mail: a-mirbagheri@ tums.ac.ir). parallel and hybrid (combination of the serial and parallel mechanisms) [6].

Each of the robot mechanisms has its own pros and cons. The serial mechanisms have easy mathematics, a large workspace and high dexterity, while their high inertial and small stiffness leads to a large end effector position error [4, 6]. Two well-known examples are phantom omni [7], and phantom premium [8] as the 6-DoF serial mechanisms. The master robot of the da Vinci system [9] is an 8-dof serial mechanism. However, there is no any published article about the workspace and dexterity of the master manipulator. A new serial structure in which a prismatic joint is responsible for the positioning, is suggested in [10] with the workspace size of $800 \times 800 \times 800 \mathrm{~mm}^{3}$. In contrast to the serial structures, the parallel mechanisms are accurate in end effector positioning due to their low inertia and high stiffness. However, their workspace and dexterity are limited and the solutions for forward and inverse kinematics are complicated $[4,6]$. As the commercial haptic interfaces with parallel mechanisms, Omega.7 [11] and Sigma.7 [12] (7-DoF), can be mentioned. A novel haptic master manipulator with 9-dof and the workspace volume size of $1.9535 \times 10^{8} \mathrm{~mm}^{3}$ is analyzed in [13]. A 7-DoF parallel mechanism is presented in [14] whose workspace size is $480 \times 450 \times 250 \mathrm{~mm}^{3}$. Hybrid mechanisms stand somewhere in the middle where provide desirable workspace and dexterity along with low end effector position error [4, 6]. In these mechanisms, positions and orientation of the end effector are usually set independently based on the two different mechanisms. In [15], a 6-DoF haptic master manipulator is introduced. The position is set with the help of two rotary and one prismatic joints. A gimbal mechanism is responsible for the orientation. A 6-DoF haptic manipulator, consisting of a R-Cube structurer and Hybrid-Spherical is proposed in [16]. Li, et al. [17], introduced an 8- DoF hybrid haptic device, including a 3-DoF serial mechanism and a 4-DoF quadruple-axial concurrent redundant mechanism.

In this paper, the kinematic of the master manipulator of Sina flex robotic telesurgery system, as a hybrid haptic interface, is analyzed. This new telesurgery system is introduced by Sina Robotics and Medical Innovators Co. [18] (Tehran, Iran) in 2017 by providing novel features for the patient side and surgeon side. The robot has an adjustable master console, modular surgical robotic arms integrated with the 3-DoF bed in the slave side [19, 20], and a novel cameraman robot, called Robolens $s_{\text {bedside. More specifically, a }}$ new 7-DoF hybrid haptic interface, including 3-DoF translation, 3-DoF orientation and 1-DoF grasp, is designed with 3-DoF force feedback. More technical detail about Sina flex robotic telesurgery system can be found in [21]. 
To investigate the performance of the master manipulator of the Sina flex robotic telesurgery system and its success in achieving the goal, a complete analysis of the robot kinematics is accomplished in this paper. To the best of our knowledge, this is the first paper about the Sina flex master manipulator, in which its forward and inverse kinematic are discussed using a novel geometrical approach and validated employing the optical tracking system, its dexterity is analyzed using the three common indices, and its performance is investigated through an in-vivo animal experiment. To this end, first, the forward and inverse kinematic of the robot are derived by using a new geometric approach. To validate the forward kinematic equations, NDI tracker is used. Then, the Jacobian of the robot is determined and accordingly, the dexterity of the robot is examined by exploiting three different indices. Finally, two real data extracted from two dog surgeries are evaluated to show the used workspace and associated dexterity during the real procedures.

In the section II, the mechanism of the master manipulator, its forward and inverse kinematic, and the Jacobian of the robot are explained. The section III is about the analysis of the robot workspace and dexterity. In the section $\mathrm{V}$, the validation overview including the simulation, experiment and animal study is explained. The results and discussion are provided in the section IV. The conclusion is drawn in the section VI.

\section{MASter Manipulator Kinematic AnAlysis}

\section{A. Mechanism Introduction}

To provide a large workspace along with a small error of the end effector position, large stiffness and small inertia, the master manipulator mechanism of Sina flex $_{\text {telesurgery system }}$ takes the advantage of a hybrid (serial and parallel) mechanism. The structure consists of a semi-parallelogram followed by a serial spherical mechanism (gimbal mechanism) (Fig. 1(a)). It has seven DoFs including three active translation DoFs provided by the semi-parallelogram mechanism used for the haptic application, three passive rotational DoFs prepared by a serial mechanism, and one active grasp DoF. The design of the master manipulator defines a remote center of motion (RCM) point which is the center of a sphere that the serial mechanism rotates about (Fig. 1(b)). The RCM point is located on the extension of the last link of the semi-parallelogram mechanism and at the intersection of spherical mechanism joints' axes. The RCM position is determined by the semi-parallelogram mechanism and dictates the desired position of the slave robot end effector. The desired orientation of the slave part is specified by the orientation of the master robot end effector made by the spherical mechanism and read by the sensors mounted to this mechanism joints.

\section{B. Kinematic Description}

As it is mentioned in sub-section A, the RCM position and the end effector orientation are the necessary parameters to be sent to the slave part. Therefore, the kinematic equations for calculating these parameters are obtained in this sub-section. First, the position of the RCM point is derived based on the semi-parallelogram mechanism, and then, the orientation of the end effector is calculated.
Figure 1. (a) The structure of the master parts and The frames defind for the kinematic equations of RCM possition, (b) The frames defined on the gimabl mechanism

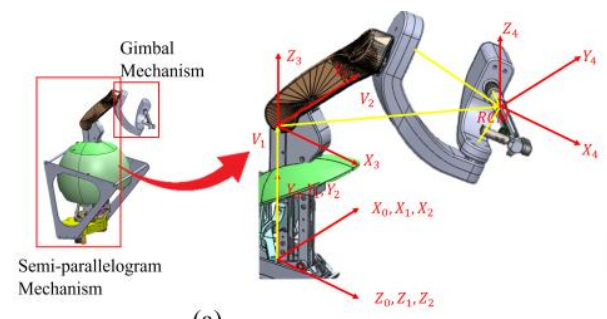

(a)

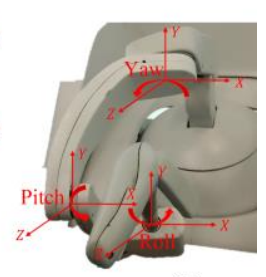

(b)
Due to the specific design of the semi-parallelogram part, a geometrical approach explained in the following is employed to derive the kinematic equations. Using this approach reduces the number of the variables in comparison to the method suggested in [22], reducing the computational cost.

The first step for the kinematic analysis, is to define the reference frame and the body frames of the manipulator. As it is shown in Fig. 1(a), the reference frame $\left(X_{0}-Y_{0}-Z_{0}\right)$ is considered on a passive joint of the semi-parallelogram. The body frames $X_{1}-Y_{1}-Z_{1}$, and $X_{2}-Y_{2}-Z_{2}$ are defined at the same point. Two vectors with constant values $\left(\overrightarrow{V_{1}}\right.$ and $\left.\overrightarrow{V_{2}}\right)$ are used to define the next body frames $X_{3}-Y_{3}-Z_{3}$ and $X_{4}-Y_{4}-Z_{4}$ (Fig. 1(a)). The values of $\overrightarrow{V_{1}}$ and $\overrightarrow{V_{2}}$ are measured using the CAD files.

In the next step, the transformation matrices between the frames are derived using the proposed geometric approach.

$$
\begin{gathered}
{ }^{0} T_{3}=\left[\begin{array}{cc}
\underbrace{\operatorname{Rot}\left(Y_{0}, \text { pan }\right)} & 0_{3 \times 1} \\
{ }^{0} R_{1} & 1
\end{array}\right]\left[\begin{array}{cc}
{\operatorname{Rot}\left(X_{1}, \text { tilt }_{1}\right)}_{{ }_{R_{2}}}^{{ }^{1}} & 0_{3 \times 1} \\
0 & 1
\end{array}\right]\left[\begin{array}{c}
\overrightarrow{V_{1}} \\
1
\end{array}\right] \\
{ }^{0} T_{4}={ }^{0} T_{3}\left[\begin{array}{cc}
{ }^{\operatorname{Rot}\left(X_{3},\left(\text { tilt }_{2}-\text { tilt }_{1}\right)\right.} & 0_{3 \times 1} \\
{ }^{3} R_{4} & 1
\end{array}\right]\left[\begin{array}{c}
\overrightarrow{V_{2}} \\
1
\end{array}\right]
\end{gathered}
$$

where, pan , tilt $_{1}$ and tilt $_{2}$ are the angles defined in the semi-parallelogram mechanism where the rotations about them provide the translation DoF and set the RCM position.

Finally, by merging (1) and (2), the RCM position $\left(p_{\text {RCM }}\right)$ can be calculated as:

$$
p_{R C M}=\left[\begin{array}{llll}
x_{R C M} & y_{R C M} & z_{R C M} & 1
\end{array}\right]^{T}={ }^{0} T_{2}\left(\left[\begin{array}{c}
\overrightarrow{V_{1}} \\
1
\end{array}\right]+{ }^{3} T_{4}\right)
$$

Now, the method for calculating the end effector orientation is described. To this end, as for the semiparallelogram part, a geometrical approach is employed instead of the conventional Denvit Hartenberg method to reduce the computational cost. The body frames, parallel to the reference frame, are defined at each gimbal joint as it is shown in Fig. 1(b). Since the axes of the joints deviate from the defined frame axes, two rotations introduced in (4) are needed to represent these joints in the body frames. 


$$
R_{G}=\operatorname{Rot}\left(X, \theta_{1}\right) \operatorname{Rot}\left(Y, \theta_{2}\right)
$$

It can be seen in Fig. 1(b), the orientation of the gimbal end effector with respect to the RCM is determined by applying three rotations as $\operatorname{Rot}(Z, y a w), \operatorname{Rot}(X$, pitch $)$ and $\operatorname{Rot}(Y, r o l l)$ about the three joints, respectively. Therefore, the final orientation of the end effector can be obtained as:

$$
\begin{gathered}
R_{G}=\operatorname{Rot}\left(X, \theta_{1}\right) \operatorname{Rot}\left(Y, \theta_{2}\right) \\
R_{O}=\operatorname{Rot}(Z, \text { yaw }) \operatorname{Rot}(X, \text { pitch }) \operatorname{Rot}(Y, \text { roll }) \\
{ }^{0} R_{E E}=\underbrace{R_{R C M}}_{{ }^{0} R_{1}{ }^{1} R_{2}{ }^{3} R_{4}} R_{G} R_{O}
\end{gathered}
$$

\section{Inverse Kinematic}

The inverse kinematic derivation is necessary for homing the master robot. the three joints of the semi-parallelogram are active, which are used for determining the home position of the robot. Then, the inverse kinematic for obtaining the active angles based on the RCM position are calculated.

In the Fig. 2(a), assume that $p_{R C M 0}, \overrightarrow{V_{1}}$ and $\overrightarrow{V_{2}}$ (the vectors defined in section II-B) belongs to the robot home position, and $p_{R C M}, \vec{V}_{p_{1}}$ and $\vec{V}_{p_{2}}$ belongs to the current position. Therefore, tilt $_{2}=$ tilt $_{2}^{\text {auxp }}-$ tilt $_{2}^{\text {aux }}$.

Exploiting the cosine rule, tilt $_{p}^{\text {aux } 2}$ and tilt $^{\text {auxz }}$ can be calculated as:

$$
\begin{aligned}
\text { tilt }_{2}^{\text {aux }} & =\cos ^{-1}\left(\left(\left|\overrightarrow{V_{1}}\right|^{2}+\left|\overrightarrow{V_{2}}\right|^{2}-\left|\vec{p}_{R C M 0}\right|^{2}\right) / 2\left|\overrightarrow{V_{1}}\right|\left|\overrightarrow{V_{2}}\right|\right) \\
t_{i l l} t_{2}^{\text {auxp }} & =\cos ^{-1}\left(\left(\left|\overrightarrow{V_{p_{1}}}\right|^{2}+\left|\overrightarrow{V_{p_{n}}}\right|^{2}-\left|\vec{p}_{R C M}\right|^{2}\right) / 2\left|\overrightarrow{V_{p_{1}}}\right|\left|\overrightarrow{V_{p_{n}}}\right|\right)
\end{aligned}
$$

Similarly, tilt $_{1}$ is calculated using Fig. 2(b) and the sine rule as:

$$
\begin{gathered}
\text { tilt }_{1}=\text { tilt }_{1}^{\text {auxp }}-\text { tilt }_{1}^{\text {aux }} \\
\text { tilt }_{1}^{\text {aux }}=\tan ^{-1}\left(y_{R C M} / x_{R C M}\right) \\
\text { tilt }_{1}^{\text {auxp }}=\sin ^{-1}\left(\left(\left|{\overrightarrow{V_{p_{2}}}}^{2}\right|^{2} \sin \left(\text { tilt }_{2}^{\text {auxp }}\right)\right) /\left|\vec{p}_{R C M}\right|^{2}\right)
\end{gathered}
$$

Finally, pan is obtained using the components of $\vec{p}_{R C M}$ in $Z$ and $X$ directions as:

$$
\text { pan }=\tan ^{-1}\left(x_{R C M} / z_{R C M}\right)
$$

Figure 2. The vectors defined to (a) show the home and current positions to calculate tilt $_{2}^{\text {auxp }}$, (b) calculate tilt $_{1}^{\text {auxp }}$

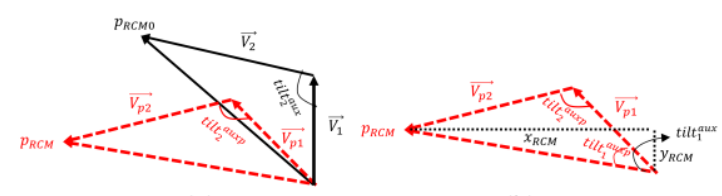

(a)

(b)

\section{Jacobian}

Jacobian derivation is a crucial task for each robot to be used for further either kinematic or dynamic analysis and the robot control [23]. This matrix is composed of two parts, $I_{v}$ and $J_{\omega}$, which relates the linear and angular velocities of the end effector, respectively, with joints angular velocities $(\dot{q})$.

Since the handles of the master console can be considered as reconfigurable robots, the method presented in [23] is used for calculating the Jacobian. In this method, $I_{v}$ and $J_{\omega}$ are obtained independently. To this end, the homogenous transformation matrix of the master manipulator for the handle end effector with respect to the defined reference frame is used [22].

\section{WORKSPACE AND DEXTERITY}

\section{A. Workspace}

Since the position and orientation of the slave robot is dictated by the master robot, to have access to all the desire points in patient side, the surgeon side workspace should be large enough. To derive the robot workspace, the RCM positions, the end effector positions and orientations are calculated for different values of joint angles in their range of motions. The position of the end effector is calculated using the RCM position and the distance between the RCM and end effector as the radius of the spherical mechanism $\left(r_{s}\right)$.

\section{B. Dexterity}

For the robots' dexterity analysis, various indices are defined in the literature including the inverse of the condition number $\left(I_{C}\right)$, manipulability $\left(I_{M}\right)$, and minimum singular value $\left(I_{S}\right)[24]$. The definition of each of these indices are as:

$$
I_{C}=\frac{1}{k}, k=\frac{\sigma_{\max }}{\sigma_{\min }}, I_{M}=\sqrt{\left|J J^{T}\right|}, I_{s}=\sigma_{\min }
$$

where, $\sigma$ indicates the singular value of $J$.

The inverse condition number lies between 0 and 1, where the values closer to 1 shows the more dexterous robot which is far away from the singular points. The manipulability index has some problems including order dependency, unit (8) dependency and scale dependency, which makes it difficult to compare the manipulability of different robots. As a solution, the normalized mobility index, as it is defined in (11), is suggested in [25]. In this equation, $I_{M_{i}}, i=1, \ldots, r$ is the manipulability index at $i^{\text {th }}$ workspace point and $r$ is the number of workspace points where the manipulability is calculated

$$
I_{M_{n}}=\frac{I_{M_{1} \ldots I_{M_{r}}}}{\max \left(I_{M_{1}} \ldots I_{M_{r}}\right)}
$$

The minimum singular value can show how much the Jacobian determinate is close to zero and singular points.

In this paper, all of these indices are calculated and the results are compared. For each point of the end effector in its workspace, the Jacobian is also calculated. Then, using the singular value decomposition method, the singular values are 
derived. Accordingly, the so-called indices are obtained. Besides, to investigate both of the position and orientation singularities as defined in [26], each index is calculated for both parts of the Jacobian, $J_{v}$ and $J_{\omega}$.

As the indices are calculated discreetly for the points of the workspace, to analyze the dexterity of the robot for the whole workspace, Global Conditioning Index $(\widetilde{G C} I)$ and Global Mobility Index $(\widehat{G M I})$ are calculated discreetly for $N$ points as:

$$
\widetilde{G C I}=\sum_{i=1}^{N} I_{C} / N, \widehat{G M I}=\sum_{i=1}^{N} I_{M_{n}} / N
$$

\section{VALIDATION OVERVIEW}

\section{A. Simulation}

To evaluate the proposed method, simulation in MALAB 2019b (MathWorks, MA) is employed. The reachable workspace of each master manipulator end effector is calculated for discrete angles of pan , tilt $_{1}$ and tilt $_{2}$ in their range of motion with $1,0.4$, and 0.04 degrees increments, respectively. To analyze the correctness of the inverse kinematic equation, several RCM positions are derived by assigning the discrete values to pan, tilt $_{1}$ and tilt $_{2}$, as before, and solving (3). These RCM positions are given to (7), (8) and (9) to calculate the related pan, tilt $_{1}$ and tilt $_{2}$ angles. Then, these angles are compared with the ones used to obtain the RCM positions. The dexterity of the robot at each workspace point is calculated using (10) and (11) for $I_{C}, I_{M_{n}}$ and $I_{S}$. To do this, $I_{v}$ is used to calculate the indices. Furthermore, $\widetilde{G C I}$ and $\overparen{G M I}$ are calculated for 508,200 workspace points.

\section{B. Experimental Validation}

Ensuring the proposed method for the direct kinematic of the robot is correct, a validation test is introduced. For the test, NDI tracker and passive markers are used. The set-up of the experimental test is shown in Fig. 3(a). The markers are installed near the RCM as it is shown in Fig. 3(b), and their positions $\left({ }^{T r} p_{\text {marker }}\right)$ and orientations $\left({ }^{T r} R_{\text {Marker }}\right)$ are tracked and provided using the camera of the NDI tracker. To verify the kinematic equations, the trajectory of the RCM positions calculated by the kinematic equations $\left({ }^{R o b} p_{R C M}\right)$ is compared with the values derived using the markers information $\left({ }^{R o b} p_{R C M}^{\prime}\right)$ as:

$$
\begin{gathered}
{ }^{R o b} R_{T r}={ }^{R o b} R_{R C M}{ }^{R C M} R_{\text {Marker }}{ }^{\text {Marker }} R_{T r} \\
{ }^{T r} p_{R C M}={ }^{T r} p_{\text {marker }}+{ }^{T r} R_{\text {Marker }}{ }^{\text {Marker }} p_{R C M}
\end{gathered}
$$

where ${ }^{R o b} R_{R C M}$ is the rotation matrix of the RCM frame with respect to the robot frame and is equal to the identity matrix, ${ }^{R C M} R_{\text {Marker }}$ is the rotation matrix of the Marker with respect to the RCM frame and is considered as $\operatorname{Rot}\left(y,-\frac{\pi}{2}\right) \operatorname{Rot}\left(x, \frac{\pi}{2}\right)$, and ${ }^{\text {Marker }} p_{R C M}$ is the position of the RCM in the Marker frame. To derive ${ }^{R o b} T_{T r}$, the robot is kept still. Since in such situation, ${ }^{R o b} p_{R C M}$ and ${ }^{R o b} p_{R C M}^{\prime}$ should be zero in any discretion, the existed offsets show ${ }^{R o b} T_{T r}$.

\section{Animal Study}

To analyze the size of the workspace and the robot dexterity during a real surgery, the data related to a vasectomy robotic surgery by Sina flex $_{\text {telesurgery system }}$ done on a 90-month dog with $30 \mathrm{~kg}$ weight and $110 \mathrm{~cm}$ length are analyzed (Fig. 4). The operation in the experiment completed by following the General Guide to Ethics in Medical Research obtained from Iran policy council of the ministry of health and medical education. The procedure was performed in a sterilized environment at Iran Advanced Clinical Training (iACT) Center, Imam Khomeini Hospital complex for the animal, while the main surgeon was at Sina hospital where is seven kilometers far away from the iACT center. The steps for preparing the animal, robot and performing the surgery were accomplished according to Preclinical study of Sina robotic surgery system research plan approved by Tehran University of Medical Sciences. The time of the vasectomy surgery, complications of the surgery, adverse events, the success percentage of the operations along with the kinematic parameters of the master manipulators and slave arms were recorded.

\section{RESUlTS AND DiscUSSION}

The reachable workspace of the robot end effector with respect to the reference frame is demonstrated in Fig. 5. According to this Fig, the reachable workspace for the master manipulator end effector is $600 \times 571 \times 560 \mathrm{~mm}^{3}$. This large workspace is about 40 times greater than the ergonomic human hand workspace [10] which guarantees an enough shared workspace.

The results of the inverse kinematic method verification are shown Fig. 6. As only the first three joints of the handle are active, the inverse kinematic is derived for the semiparallelogram mechanisms. As it is clear from Fig. 6., the error for the pan, tilt $_{1}$ and tilt $_{2}$ angles is zero which shows the accuracy of the inverse kinematic equations.

Figure 3. (a) The experimental set-up, (b) the markers attached to the gimbal handle.

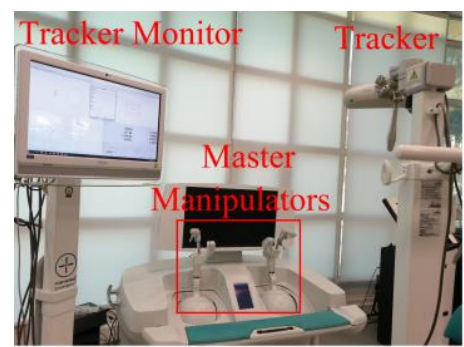

(a)

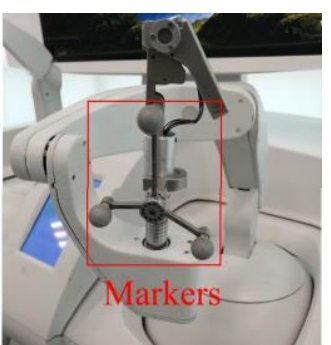

(b)
Figure 4. The vasectomy surgery on a dog using Sinaflex robotic telesurgery system.
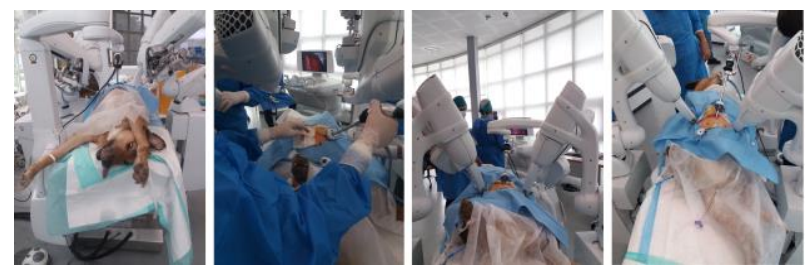
Figure 5. The reachable workspce of the master manipulator end effector with respect to the refernce frame.

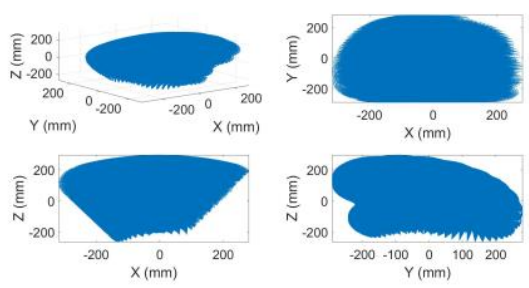

The dexterity of the robot in getting various positions for each of the indices defined in section III-B, is derived for each of the end effector workspace point and shown in Fig. 7. This figure reveals the dexterity of the robot in reaching the various positions. The results are summarized in the first row of Table I.

Fig. 8. shows the trajectory derived by the equation in comparison with the one come from the experimental test explained in section IV-B. To check the authenticity of the forward kinematic equations, the trajectory of the marker attached to the RCM (red) is compared with RCM trajectory obtained by the equations (blue) in Fig. 7. As it can be seen, the marker and the RCM follow the same trajectories. The differences between the positions come from the difference between the real position of the marker and the RCM position. Besides, the vibration of the markers with respect to the handle, which is made due to some lack of rigidity, can cause some position errors.

The animal study shows that the operational workspace is as large as $136 \times 197 \times 186 \mathrm{~mm}$ and $138 \times 226 \times 208 \mathrm{~mm}$ for the left and right master manipulators, respectively (Fig. 9). The dexterity indices values derived from the simulation and animal study (Fig. 9) are compared in TABLE I. As it can be inferred from this table, the robot provides high dexterity for the operational workspace with $\widetilde{G C I}=0.6565$ and $\widehat{G M I}=0.7628$. The performance of the Sina flex master manipulator is investigated in compared with some of proposed master manipulator/haptic interfaces by the other researchers [10, 13, 15, 27-29] in TABLE II. Based on this table, the dexterity of the proposed master manipulator is comparable with the ones presented in $[15,27,28]$.

However, the workspace of Sina $\mathrm{flex}_{\text {master manipulator }}$ outperforms their workspace. A large workspace and high dexterity of the novel master manipulator of the SINA $_{\text {flex }}$ telesurgery system makes it an appropriate platform for accomplishing robotic surgery tasks.

Figure 6. The comparison between the real pan, tilt $t_{1}$ and tilt $_{2}$ angles and the ones derived by the the inverse kinematics.

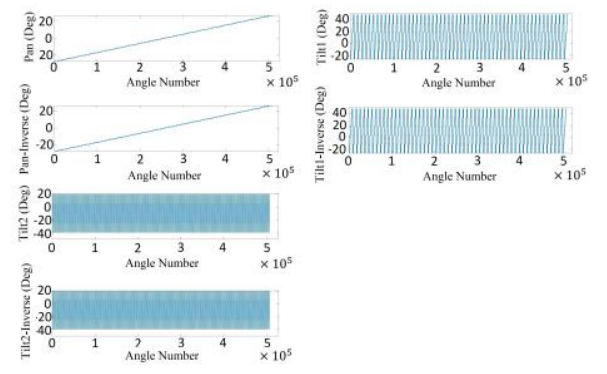

Figure 7. The dexterity of the master robot based on: (a) inverse condition number,(b) manipulability, (c) minimum singular value.

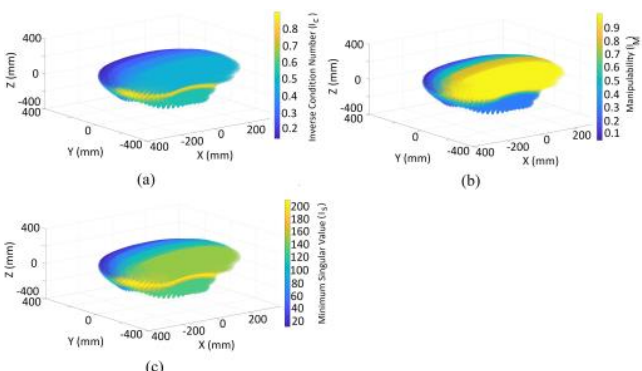

TABLE I. THE DEXTERITY OF THE ROBOT DERIVED FROM THE SIMULATION AND ANIMAL STUDY.

\begin{tabular}{|c|c|c|c|c|c|}
\hline \multirow[b]{2}{*}{ Dexterity Index } & \multirow[b]{2}{*}{$I_{C}$} & $\operatorname{Max}$ & \multirow[b]{2}{*}{$I_{M}$} & Max & \multirow[b]{2}{*}{$\operatorname{Min}\left(I_{S}\right)$} \\
\hline & & $\frac{\text { Min }}{\widetilde{G C I}}$ & & $\frac{\text { Min }}{\overline{G M I}}$ & \\
\hline \multirow{3}{*}{ Simulation: $I_{v}$} & \multicolumn{2}{|c|}{0.8963} & \multicolumn{2}{|c|}{1} & \multirow{3}{*}{10.6193} \\
\hline & \multirow{2}{*}{\multicolumn{2}{|c|}{$\begin{array}{l}0.0331 \\
0.4557\end{array}$}} & \multicolumn{2}{|c|}{0.0372} & \\
\hline & & & 0.54 & & \\
\hline \multirow{3}{*}{ Animal: $J_{v}$} & \multicolumn{2}{|c|}{0.8953} & \multicolumn{2}{|c|}{1} & \multirow{3}{*}{42.0563} \\
\hline & \multirow{2}{*}{\multicolumn{2}{|c|}{$\begin{array}{l}0.1348 \\
0.6565\end{array}$}} & \multicolumn{2}{|c|}{0.0392} & \\
\hline & & & \multicolumn{2}{|c|}{0.7628} & \\
\hline
\end{tabular}

Figure 8. The trajectory derived by (3) (blue) in compared with the one comes from the experiment (red).

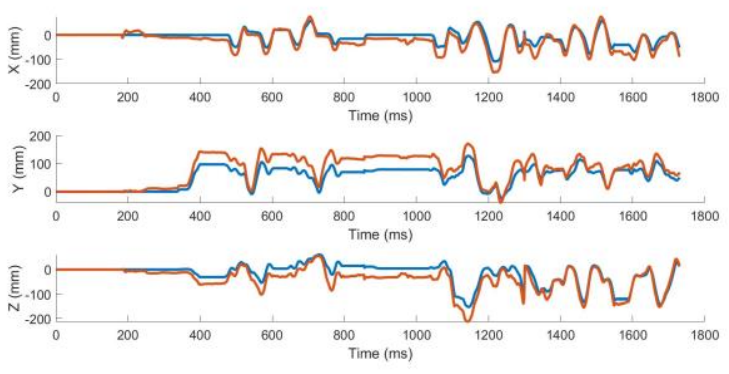

Figure 9. The workspace of the master manipulator end effector for the animal study: a) left handle, b) right handle.

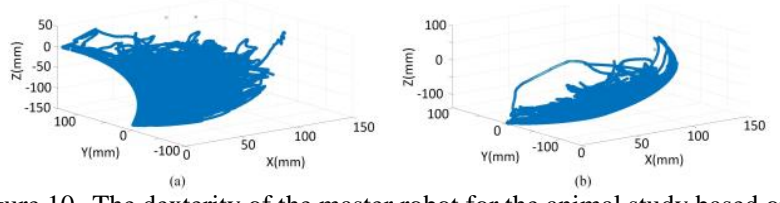

Figure 10. The dexterity of the master robot for the animal study based on: (a) inverse condition number, (b) manipulability, (c) minimum singular value.

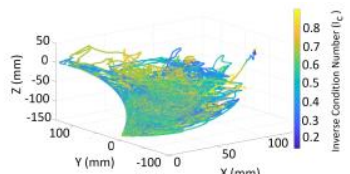

(a)

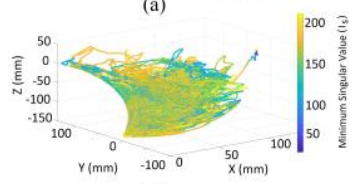

(c)

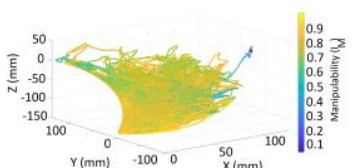

(b) 


\section{CONCLUSION}

In this paper, the forward and inverse kinematic of the Sina $_{\text {flex }}$ master manipulator is derived using a new geometrical approach and the validation is done using theoretical equations and the experiment. Then, the workspace of the robot is investigated. The robot dexterity is analyzed using the three Jacobian based indices including the manipulability, inverse condition number and the minimum singular value. To analyze the robot dexterity during a real surgery, an animal study is conducted. According to the results, the inverse condition number and manipulability indices for 508,200 points of the $600 \times 571 \times 560 \mathrm{~mm}^{3}$ workspace are 0.4557 and 0.5432 , respectively. These values are as 0.6565 and 0.7628 for the operational workspace. These results prove the large and dexterous workspace of the Sina flex master manipulator, which guarantees its potential for future human surgery as a commercial robotic telesurgery system.

TABLE II. THE COMPARISON OF THE WORKSPACE AND DEXTERITY OF HAPTIC INTERFACE MANIPULATORS..

\begin{tabular}{|l|l|l|}
\hline \multicolumn{1}{|c|}{ Robot } & \multicolumn{1}{|c|}{ Workspace } & \multicolumn{1}{|c|}{ Dexterity $\left(I_{v}\right)$} \\
\hline A 7-DoF serial robot [29] & NA & $\min \left(I_{s}\right)=0$ \\
\hline Stanford Manipulator [29] & NA & $\min \left(I_{s}\right)=0$ \\
\hline A proposed structure [15] & $\begin{array}{l}\phi 800 \times 230 \mathrm{~mm} \\
(\text { insertion })\end{array}$ & $G C I=0.6624$ \\
\hline Phantom Omni [15, 27] & $160 \times 120 \times 70 \mathrm{~mm}^{3}$ & $\begin{array}{l}G C I=0.4146 \\
\max \left(I_{M}\right)=0.8\end{array}$ \\
\hline $\begin{array}{l}\text { A proposed master } \\
\text { manipulator [10] }\end{array}$ & $800 \times 800 \times 800 \mathrm{~mm}^{3}$ & $I_{C}<0.2$ \\
\hline $\begin{array}{l}\text { PHANToM Desktop } \\
\text { haptic device [28] }\end{array}$ & $160 \times 120 \times 120 \mathrm{~mm}^{3}$ & $G C I=0.419$ \\
\hline $\begin{array}{l}\text { A proposed Haptic Master } \\
\text { Manipulator [13] }\end{array}$ & $1.9535 \times 10^{8} \mathrm{~mm}^{3}$ & $I_{C}<0.5$ \\
\hline
\end{tabular}

\section{REFERENCES}

E. I. George and C. T. C. J. J. J. o. t. S. o. L. S. Brand, "Origins of robotic surgery: from skepticism to standard of care," vol. 22, no. 4, 2018.

[2] K. H. Sheetz, J. Claflin, and J. B. J. J. n. o. Dimick, "Trends in the adoption of robotic surgery for common surgical procedures," vol. 3, no. 1, pp. e1918911-e1918911, 2020.

[3] C. Arenas-Gallo, J. E. Shoag, and J. C. J. U. C. Hu, "Optimizing Surgical Techniques in Robot-Assisted Radical Prostatectomy," vol. 48, no. 1, pp. 1-9, 2021.

[4] A. Torabi, A. A. Nazari, E. Conrad-Baldwin, K. Zareinia, and M. J. P. i. B. E. Tavakoli, "Kinematic design of linkage-based haptic interfaces for medical applications: a review," 2021.

[5] D. Zhang, J. Liu, A. Gao, and G.-Z. Yang, "An ergonomic shared workspace analysis framework for the optimal placement of a compact master control console," J IEEE Robotics Automation Letters, vol. 5, no. 2, pp. 2995-3002, 2020.

[6] W. Dangxiao, G. Yuan, L. Shiyi, Y. Zhang, X. Weiliang, and X. Jing, "Haptic display for virtual reality: progress and challenges," J Virtual Reality Intelligent Hardware, vol. 1, no. 2, pp. 136-162, 2019.

[7] (2021.11.17). TouchTM. Available: https://www.3dsystems.com/haptics-devices/touch

[8] (2021.11.17). Phantom PremiumTM. Available: https://www.3dsystems.com/haptics-devices/3d-systemsphantom-premium

[9] C. Freschi, V. Ferrari, F. Melfi, M. Ferrari, F. Mosca, and A. Cuschieri, "Technical review of the da Vinci surgical telemanipulator," $J$ The International Journal of Medical Robotics Computer Assisted Surgery, vol. 9, no. 4, pp. 396-406, 2013.

[10] X. Chen, X. Xin, B. Zhao, Y. He, Y. Hu, and S. Liu, "Design and analysis of a haptic master manipulator for minimally invasive surgery," in 2017 IEEE International Conference on Information and Automation (ICIA), 2017, pp. 260-265: IEEE.

[11] Force dimension Omega $7 . \quad$ Available: https://www.forcedimension.com/products/omega

[12] (2021.11.17). Force Dimension Sigma 7. Available: https://www.forcedimension.com/products/sigma

[13] Y. Liang, L. Sun, Z. Du, Z. Yan, and W. Wang, "Mechanism design and optimization of a haptic master manipulator for laparoscopic surgical robots," J IEEE Access, vol. 7, pp. 147808147824, 2019.

[14] H. Bassan, A. Talasaz, and R. V. Patel, "Design and characterization of a 7-DOF haptic interface for a minimally invasive surgery test-bed," in 2009 IEEE/RSJ International Conference on Intelligent Robots and Systems, 2009, pp. 40984103: IEEE.

[15] H. Lee, B. Cheon, M. Hwang, D. Kang, D. S. J. T. I. J. o. M. R. Kwon, and C. A. Surgery, "A master manipulator with a remote-center-of-motion kinematic structure for a minimally invasive robotic surgical system," vol. 14, no. 1, p. e1865, 2018. M. İ. C. Dede, Ö. Selvi, T. Bilgincan, and Y. Kant, "Design of a haptic device for teleoperation and virtual reality systems," in 2009 IEEE International Conference on Systems, Man and Cybernetics, 2009, pp. 3623-3628: IEEE.

[17] Y. Li, Z. Yan, H. Wang, Z. Du, and Y. Zhang, "Design and optimization of a haptic manipulator using series-parallel mechanism," in 2012 IEEE International Conference on Mechatronics and Automation, 2012, pp. 2140-2145: IEEE.

[18] (2021.3.25). Available: https://sinamed.ir/

[19] A. Alamdar, F. Farahmand, S. Behzadipour, A. J. M. Mirbagheri, and M. Theory, "A geometrical approach for configuration and singularity analysis of a new non-symmetric 2DOF 5R Spherical Parallel Manipulator," vol. 147, p. 103747 , 2020.

[20] S. Toumajpour, H. Moradi, and A. Mirbagheri, "Arrangement Optimization of Robotic surgery Arms at Sina flex surgical platform," in 2021 9th RSI International Conference on Robotics and Mechatronics (ICRoM), 2021, pp. 542-547: IEEE.

M. H. Abedin-Nasab, Handbook of robotic and image-guided surgery. Elsevier, 2019.

M. Aghanouri, P. Kheradmand, M. Mousavi, H. Moradi, and A. Mirbagheri, "Kinematic and Workspace Analysis of the Master Robot in the Sina flex Robotic Telesurgery System," in Annual International Conference of the IEEE Engineering in Medicine and Biology Society. IEEE Engineering in Medicine and Biology Society. Annual International Conference, 2021, vol. 2021, pp. 4777-4780.

[23] P. Chembrammel and T. Kesavadas, "A new implementation for online calculation of manipulator Jacobian," J PloS one, vol. 14, no. 2, p. e0212018, 2019.

[24] J.-P. Merlet, "Jacobian, manipulability, condition number, and accuracy of parallel robots," 2006.

[25] T. Tanev, B. J. P. o. e. c. Stoyanov, and robotics, "On the performance indexes for robot manipulators," vol. 49, pp. 64-71, 2000.

[26] I. Zaplana, H. Hadfield, and J. J. a. p. a. Lasenby, "Singularities of serial robots: Identification and distance computation using geometric algebra," 2021.

[27] J. San Martín and G. Triviño, "A study of the Manipulability of the PHANToMTM OMNITM Haptic Interface."

[28] Y. Maddahi et al., "Performance evaluation of a surgical telerobotic system using kinematic indices of the master handcontroller," in International Conference on Human Haptic Sensing and Touch Enabled Computer Applications, 2014, pp. 167-175: Springer.

[29] X. Zhang, B. Fan, C. Wang, and X. J. E. Cheng, "Analysis of Singular Configuration of Robotic Manipulators," vol. 10, no. 18 , p. 2189, 2021. 